\title{
Evaluating The Temporal Relationship Between Climatic and Demographic Events Using Radiocarbon Data: A Case-Study From The Chulmun Period, Korea
}

Habeom Kim

University of Oregon

Gyoung-Ah Lee

University of Oregon

Enrico Ryunosuke Crema ( $\nabla$ erc62@cam.ac.uk)

University of Cambridge

\section{Research Article}

Keywords: temporal relationship, climatic, demographic, radiocarbon

Posted Date: October 5th, 2021

DOl: https://doi.org/10.21203/rs.3.rs-944473/v1

License: (c) (i) This work is licensed under a Creative Commons Attribution 4.0 International License.

Read Full License 


\section{Abstract}

We investigate the relationship between climatic and demographic events in Korea during the Chulmun period $\left(10,000-3,500 \mathrm{cal}\right.$. BP) by analyzing paleoenvironmental proxies and ${ }^{14} \mathrm{C}$ dates. We focus on testing whether a cooling climate, and its potential impact on millet productivity around 4,500 cal. BP triggered the population decline observed in the archaeological record. We employ a Bayesian approach that measures the temporal relationship between climatic events and change-points in the rate of growth in human population as inferred by radiocarbon density. Our results do not support the climate-induced population decline hypothesis for three reasons. First, we could not determine that the cooling climatic event necessarily preceded the reversal point in population growth rate as inferred by the radiocarbon record. Second, we did not find evidence showing a significant reduction of millet-associated dates occurring during the cooling climate. Third, we detected a spatially differentiated pattern of decline in inland and coastal regions, indicating that the cooling climate did not impact all populations equally. We highlighted the long tradition of mobility-based subsistence strategy in the coastal region as a potential factor contributing to the spatial differences.

\section{Introduction}

Climate change has long been at the centerpiece of explanatory models of prehistoric demographic changes ${ }^{1,2,3,4,5}$. The last decade witnessed a renewed interest in this topic due to the ongoing climate crisis and the new opportunities offered by the increasing availability of paleoenvironmental proxies and large ${ }^{14} \mathrm{C}$ datasets, used as demographic proxies ${ }^{6,7}$. Paleodemographic research based on ${ }^{14} \mathrm{C}$ datasets, in particular, has benefited from substantial growth in the number of applications, most typically involving the use of the summed probability distribution of calibrated ${ }^{14} \mathrm{C}$ dates (hereafter SPDs). Based on the assumption of a consistent correlation between the number of dates and the human population size, SPDs are now commonly utilized to examine past demographic processes ${ }^{8}$. Studies utilizing SPDs have stimulated an array of studies examining the relationship between climate changes and inferred prehistoric population changes, ranging from simple visual inspections of time-series to more complex statistical analyses and models $9,10,11,12,13,14,15,16$.

While these studies undoubtedly reveal a more nuanced picture of past human-climate relationships, SPDs and, more specifically, the assumption of 'more dates-more people' have also raised criticisms. These range from the interpretability of the proxies in relation to formation, taphonomic, and recovery processes to the wide range of statistical biases associated with the summation of ${ }^{14} \mathrm{C}$ dates ${ }^{17,18,19,20}$. One such issue is the intrinsic uncertainty associated with these curves that arises from a combination of different errors, namely, sampling, radiometric measurement, and calibration. As a result, visual inspections of SPD values should be carried out with caution, and more importantly, 'direct' statistical analyses of SPD values should be avoided as they do not account for the different forms of uncertainties associated with the ${ }^{14} \mathrm{C}$ data ${ }^{19,21,22}$. 
Environmental proxies deployed in statistical comparisons with radiocarbon time-frequencies are also affected by their own chronological uncertainties. These are generally accounted for in age-depth models but rarely integrated as part of the final correlative analyses. As a result, the temporal relationship between particular trends in the demographic and the paleoenvironmental proxies (e.g. a population drop vs a cooling event) are never formally evaluated. The consequence of this lack of formality is observed when the close temporal proximity of climatic and demographic signatures are assumed to be sufficient to argue for a specific order - most typically the climatic event before the demographic one - and ultimately interpreted as evidence of a causal relationship. While to some extent such a prior belief can be justified, the extent of chronological uncertainties still needs to be accounted for, particularly if we wish to evaluate models that consider not just the temporal order but also the temporal distance between these events. The temporal distance between demographic and climatic events can reveal far more insights about adaptive responses and time-lags rather than simplistic models of climate-induced demographic processes (see for examples ${ }^{10,11}$ ).

This study investigates the relationship between climatic and demographic events occurring during the Chulmun period $(10,000-3,500 \mathrm{cal}$. BP) in Korea by taking into account these specific challenges. Chulmun, referring to the incised decoration pottery, is a local term for the Neolithic. Chulmun is typically divided into six subphases, including the Incipient (10,000-8,000 cal. BP), Initial (8,000-6,500 cal. BP), Early (6,500-5,500 cal. BP), Middle (5,500-5,000 cal. BP), Late (5,000-4,000 cal. BP), and Final (4,0003,500 cal. BP). Taking advantage of a wealth of ${ }^{14} \mathrm{C}$ dates accumulated over recent decades due to increasing rescue excavations in Korea, several authors have already inferred the population dynamics of the Chulmun period using SPDs ${ }^{23,24,24,25}$. These studies mark an important juncture in Korean archaeology as pioneering studies that intensively utilized large available ${ }^{14} \mathrm{C}$ datasets to reconstruct population trends during the Chulmun. Although each of these works have used different data filters on the ${ }^{14} \mathrm{C}$ datasets and sometimes integrated additional population proxies such as the pithouse and settlement count (see for example ${ }^{24}$ ), they all agree on two large population trends. One is the general pattern of population growth toward the Middle Chulmun, while the other is the subsequent population toward the Late and Final Chulmun.

This study specifically focuses on the latter trend, the population decline phase toward the Late and Final Chulmun. Currently, the cause of this decline is attributed to the cooling climate occurring around 4,500 cal. BP. According to Ahn et al. ${ }^{24}$ and Ahn and Hwang ${ }^{26}$, the Chulmun population peaked by the Middle Chulmun due to the adoption of millet cultivation and sedentary lifestyle originated from the centralwestern inland region. However, the onset of cooling climate by the end of Middle Chulmun severely decreased the productivity of plant resources, particularly cultivated millets such as foxtail millet (Setaria italica) and broomcorn millet (Panicum miliaceum). Once millets stopped being a viable subsistence resource due to the deteriorating climate condition, Chulmun people abandoned their cultivation as well as the sedentary lifestyle, thereby triggering the observed population decline toward the Late and Final Chulmun. The evidential ground of this hypothesis rests on the two premises. Multiple paleoclimate proxies measured near and within the Korean peninsula indicate that a global scale cold/dry climate also 
occurred in Korea between 5,500 and 4,500 cal. BP. The number and scale of Chulmun settlements in many regions of Korea decreased in the Late/Final phase ${ }^{24}$.

The previous Chulmun population studies share a number of methodological shortcomings that make the evaluation of this hypothesis difficult. First, their methods of investigation are largely limited to the visual inspection of the trends in ${ }^{14} \mathrm{C}$ data histograms and SPDs. As far as we are aware, Oh et al. ${ }^{25}$, is the only study that applied statistical testing to examine SPDs of the Korean peninsula, although their objective was simply to determine whether the inferred demographic curve deviated from an exponential growth. Second, while such null hypothesis significance testing (NHST) approach can highlight interesting fluctuations in the inferred demographic trajectories, it does not directly evaluate the potential impact of climatic events. Third, past studies do not incorporate spatial perspectives into the overall interpretations of the ${ }^{14} \mathrm{C}$ data, thereby contributing to a unilinear narrative on the processes leading to population changes. In doing so, the past studies assume a ubiquitous process of population decline without considering the possibility that the population trend may vary by region. Fourth, despite the critical role of the decline of millet cultivation in this hypothesis, past studies often miss using the ${ }^{14} \mathrm{C}$ data available on the charred millets in their analysis on Chulmun population trends.

Here we test the hypothesis on the climate-induced Chulmun population decline by filing the methodological gaps in the previous studies. We use a ${ }^{14} \mathrm{C}$ dataset $(n=683)$ from Chulmun sites in comparison with paleoclimatic data and dates associated with charred millets, then integrate a spatial dimension. If the hypothesis on a climate-induced population decline is supported, we expect to observe the following three patterns. First, the cooling climatic event would precede the population decline. Second, the population decline would be coupled with a significant decline in the relative frequency of ${ }^{14} \mathrm{C}$ dates associated with millets. The decline of millet frequency would suggest that the population decline is closely tied with the failure of millet cultivation. Third, the rate and the timing of decline would be similar across the different regions of Korea. A regionally undifferentiated pattern of decline would validate the assumption that the hypothesized process leading to the population decline is applicable on a macro-regional scale.

To check our expectations, we first divide the ${ }^{14} \mathrm{C}$ dataset into two regional groups, coastal and inland (Fig. 1). The SPD of the two regions is in line with the pattern demonstrated in the previous studies, which indicated a pattern of population growth toward the Middle Chulmun, followed by a decline in the Late/Final phase.

This regional grouping is chosen in recognition that the subsistence practices in the two regions were different prior to the Middle Chulmun. Chulmun occupations on the coast exhibited a long history of mobility-based strategy, consisting of residential mobility, where hunter-gatherer groups seasonally moved from one locality to another for subsistence practices (in sensu Binford ${ }^{27}$ ). The inland region, on the other hand, showed a strong tradition of large-scale sedentism that emerged in the Early Chulmun and intensified toward the Middle Chulmun ${ }^{24,28}$. We consider the possibility that the mobile hunter- 
gathering tradition, prevalent in the coastal regions before the Middle Chulmun, may have lingered and contributed to regional variations in the population decline pattern toward the Late/Final phase. To check this possibility, we fit a bounded double-exponential hierarchical Bayesian model ${ }^{22}$ to the ${ }^{14} \mathrm{C}$ frequency data of the two regions. Fitted parameters of the double-exponential model provide the estimated rates of growth in the two regions, as well as the timing of when the population trend reversed from positive to negative growth rates. By comparing the fitted parameter to the timing of the abrupt cooling event based on two pollen sequences ${ }^{29,30}$ and the alkenone-based sea surface temperature reconstruction ${ }^{31}$, we aim to obtain a probabilistic estimate of the temporal order between a demographic decline and a cooling event. Finally, we compare the SPDs of all Chulmun dates against that of the dates associated with millet using the Mark-permutation test ${ }^{32}$ to determine whether and when the relative frequency of milletassociated dates changed over our window of analyses.

\section{Results}

\section{Regional variations in Chulmun population trajectory: Coastal vs Inland}

Figure 2 (see also Fig. S12) shows the population growth and decline rates in the two regions before and after the change point of population trend as obtained by fitting the SPDs into the discrete doubleexponential hierarchical Bayesian model. We define the change point as a point in time when the overall population trend reversed from a growth into a decline. The median growth and decline rates in the inland region are approx. $0.18 \%$ and $-0.14 \%$, whereas they are approx. $0.06 \%$ and $-0.05 \%$ in the coastal region. The rate of past population changes in both regions is within the range of changes seen in other case studies on past populations ${ }^{22,33,34}$.

The result of our analysis opposes one of the expectations in the climate-induced population decline hypothesis. Despite its assumption in the ubiquitous applicability of the hypothesized process leading to the population decline, there is a clear spatially differentiated pattern of decline when the inland and the coastal regions are compared (see also Fig. S1). During the decline phase after the change point, the population in the inland region decreases roughly at a rate 2.5 times greater than that of the coast. Also, the median posterior change point from the growing to declining phase indicates a delayed shift in the inland sites roughly by 200 years. We note, however, that the $90 \%$ high probability density (HPD) intervals overlap, primarily due to the higher uncertainty of the estimate for the coastal region (Fig. 2, see also Fig. S12). This overlap leaves a small possibility that the posterior changepoint in both regions is concurrent rather than distanced temporally.

\section{Cooling climate in relation to the timing of the change point}

The temporal relationship between the changepoints and the cooling events shows some conflicting results. There is strong evidence that cooling events inferred from a drop in the ratio of arboreal to total pollen occurred after the changepoint observed in the frequency of ${ }^{14} \mathrm{C}$ dates with probabilities 
systematically above 0.95 (events $f$ and $g$ in Fig. 3). The median posterior indicates a gap of 200 to 800 years between the cooling events and the change point. For these two cooling events, no regional variabilities are detected. Both inland and coastal regions show a high probability that the cooling climate occurred after the population changepoint.

The alkenone-derived estimate of sea surface temperatures shows a much closer temporal distance between the cooling event and the changepoint in the fitted double-exponential model for the two regions (event $d$ in Fig. 3). The $90 \%$ HPD of the difference in the dates cover both positive and negative values, hence suggesting that we cannot dismiss the possibility that the cooling occurred before the decline in the density of ${ }^{14} \mathrm{C}$ dates. For this cooling event, a moderate regional variability in the temporal relationship is observed, with the probability that the beginning of the cooling event occurred after the population changepoint slightly higher in the coast than in the inland (event $d_{1}-C_{\text {coastal }}$ and $d_{1}-C_{\text {inland }}$ in Fig. 3).

The premise of the climate-induced population decline hypothesis rests on the cooling climate occurring before the population changepoint. This premise has not been met in our analysis involving three distinct paleoclimatic proxies. Instead, two out of the three paleoclimatic proxies confirmed the opposite is highly probable: the cooling climate occurred after the population changepoint. The analysis based on the remaining paleoclimatic proxy based on the data from Kim et al. ${ }^{31}$ yielded an inconclusive result neither confirming nor rejecting the expectation of the hypothesis.

\section{Mark-permutation test of millet-associated dates}

The Mark-permutation test between dates associated with millets and all dates yielded a P-value of 0.63 indicating that the frequencies of millet-associated dates have not changed significantly relative to the overall frequency changes of the Chulmun absolute dates (Fig. 4). While it is worth taking into consideration the small sample size, the lack of significant reduction in the frequency of millet-associated dates does not currently provide evidence to support the claim that the failure of millet farming had occurred amidst the on-going climate changes in the Late Chulmun.

\section{Discussion}

One caveat needs to be considered before we evaluate the climate-induced population decline hypothesis. We used a significant drop in the paleoclimate proxy data as a reference point when the cooling climate impacted the Chulmun population. In doing so, we effectively treated the cooling climate as a one-time event. However, as multiple paleoclimatic proxy data indicate, the cooling climate was a condition that continuously impacted the Chulmun population for at least a few hundred years between 5,500 and 4,500 cal. BP. This, however, does not necessarily prove that the cooling climate was the primary cause for the decline of Chulmun population. We emphasize that our test is focused on assessing the feasibility of the hypothesized process, where the cooling climate directly triggered a decline in the population trend. 
Accounting for the limitation of our analyses mentioned, we still remain positive that the cooling climate alone could not be the direct cause of the population decline in the Late and Final Chulmun. If the cooling climate has been directly responsible for triggering the Chulmun population decline, the population changepoint from growth to decline would have clearly occurred after a major cooling signal. However, we could not determine that the cooling climate necessarily preceded the population changepoint. On the contrary, two out of the three paleoclimatic proxy data employed here indicate that the likelihood of the population changepoint prior to the cooling climatic event is much higher than the opposite. Also, if the failure of millet cultivation has been one of the effects and contributing factors to the population decline, we would be able to detect a significant decline in the millet-associated dates before or during the Late and Final Chulmun. However, our test indicates that the frequency of millet-associated dates relative to all other ${ }^{14} \mathrm{C}$ dates did not decrease significantly either in the Late or Final phase.

Another factor adding weight against the climate-induced population decline hypothesis is the ecology of millets. The dominant millet taxa during Chulmun, foxtail and broomcorn, are known to be highly tolerant species that can grow in various conditions including drought-prone cold environments. Both species are fast-growing grain crops, and can adapt to almost all types of soil conditions ${ }^{35}$. Broomcorn millet has one of the lowest water requirements of all cereals, requiring only an annual rainfall of $200-450 \mathrm{~mm}^{36}$. Foxtail millet can grow even less than $125 \mathrm{~mm}$ rainfall in semi-arid areas during the months of growth ${ }^{37}$. Broomcorn and foxtail can mature as short as 45 and 90 days respectively and have a large temperature tolerance. Such minimum requirements would have facilitated Early Holocene people in northern China to grow them without much effort as early as 10,000 cal. BP and spread widely throughout East Asia, Far Eastern Russia, and Europe $\mathrm{B}^{38,39}$.

This study also finds that, while both inland and coastal regions experience a general population decline in the Late/Final phase, the rate of decline is much greater in the inland region than the coast. This regional variability hints at the spatial processes at work during the population decline phase in the Late/Final Chulmun. The coastal region distinguishes itself from the inland region by its long tradition in mobile subsistence strategy. Here, we consider the possibility that the mobile subsistence tradition in the coastal region may have contributed to the observed regional variations in the population decline trend.

The evidence for the residential mobility in the coastal region is found in some of the earliest Chulmun occupations. These occupations include occupations such as the Gosanri site in the coast of Jeju dating as early as 10,000 cal. BP, marking the earliest pottery culture in Korea ${ }^{40}$. Gosanri site is believed to have been used as a task-specific site seasonally used for the lithic tool production ${ }^{40}$. Other occupations reflecting residential mobility are Osanri $C$ and Munamri on the east coast dating to as early as 8,000 cal. $\mathrm{BP}$, marking the start of the Initial Chulmun ${ }^{41}$. Almost concurrently, seasonal dwellings appeared along the southern coast as evidenced in the sites such as Tongsamdong, Bibongri, Beombang sites ${ }^{41}$. The Chulmun settlers in the coastal region utilized diverse seasonal resources, both marine and terrestrial catch as well as millets, and probably used these sites as seasonal base camps rather than permanent settlements reflecting their residential mobility $28,40,42$. They practiced a 'broad-spectrum economy' 
procuring a diverse array of marine and terrestrial resources by hunting, gathering, fishing, and millet cultivation $^{42,43}$.

The coastal community experiences a major subsistence transition onset of the Middle Chulmun. From the inland region of the central-western valley, a new subsistence tradition expanded to the rest of the peninsula, penetrating into the coast, in a phenomenon recognized by some as the 'Middle Chulmun expansion ${ }^{24,28}$. While the new tradition is still largely grounded in the preceding phase's broad-spectrum economy, it was newly paired with the beginning of large-scale sedentism. The most widely cited example of the new tradition is the Amsadong site in the Han River valley approximately $50 \mathrm{~km}$ inland from the coast. Amsadong yielded dozens of pit-houses and outdoor structures where food production and processing tools were found, including digging tools, reaping knives, and grinding slabs ${ }^{44}$. These sedentary communities are popularly regarded as early food producers practicing millet cultivation ${ }^{24,28}$. However, the practice of small-scale food production was not confined to the new sedentary communities as the coastal communities also utilized plant resources, including millet and azuki bean, as early as the Initial Chulmun ${ }^{42,43}$.

The conventional view on the Chulmun cultural trajectory accepts that the new sedentary way of life largely replaced the existing mobile tradition following the Middle Chulmun expansion. Indeed this view seems to be supported by archaeological findings, including the ubiquitous popularization of the 'combmarked' style pottery associated with the sedentary communities and new appearance of large-scale villages. This view contends, however, that the Chulmun villagers in the Late phase eventually abandoned the sedentism and readopted the mobility-based lifestyle consisting of logistical mobility mixed with some degree of residential mobility $24,26,45$. The evidence for this view is attributed to the decline in the number of settlements and the increase of outdoor features, including open-air hearths and shell middens ${ }^{24,26}$.

The population decline inferred by the deterioration of sedentism in the Late/Final phase is an observable phenomenon as suggested by the previous studies ${ }^{24,26}$. It is beyond the scope of this study to ascertain the primary factor for the sedentism decline, although our study suggests the cooling climate alone cannot sufficiently explain the cause of the decline. What is certain, however, is that the coastal Chulmun communities experienced a much moderate rate of decline than the inland. What differences in the coastal and the inland region caused this pattern?

The differences may lie in the fact that, unlike the inland, the coastal region has a deep tradition of practicing mobility-based subsistence strategy over thousands of years. It is possible that the sedentary lifestyle popularized after the Middle Chulmun expansion may not have completely replaced the coast's mobility-based subsistence tradition that has been practiced over hundreds of generations. Instead, the traditional ecological knowledge (TEK) that the coastal community accumulated over the long term may have been inherited and practiced to some extent in the Late/Final phase even after the Middle Chulmun expansion. 
TEK refers to a cumulative body of knowledge and practices about the relationships of living beings with one another and their physical environment evolved by adaptive processes and inherited by cultural transmission ${ }^{46}$. TEK may have provided relative resilience to the coastal communities by diversifying their subsistence strategies during a time when the overall population was declining due to the deteriorating sedentary way of life. TEK may therefore account for the reasons why the sharp decline observed in the inland regions during the Late/Final phase is not seen in the coast.

Concluding the discussions on Chulmun population change, we point out that Chulmun is not a unique case where climate change does not play a causative role in triggering a population response. Many case studies worldwide reveal a nuanced relationship between the population and the climate, where the change in the former is impacted by the latter in a complex multitude of ways rather than in a deterministic one-way fashion. For example, Shennan et al. ${ }^{9}$ investigate the relationship between population and climate in Central and Northwest Europe during the mid-Holocene by computing crosscorrelations between several climatic proxies and the SPDs. Their findings indicate that the variations in population levels are largely not associated with climatic changes. In a similar vein, DiNapoli et al. ${ }^{47}$ observe the population trend of Rapa Nui between the time of initial human settlement (ca. $800 \mathrm{cal}$. BP) and the period shortly after the European contact using Approximate Bayseian Computation method. Their result highlights the resilience of the island's population, reflected in the steady population levels on the island until the timing of European arrival. This finding sharply contests the previous study's claim that climate-induced drought resulted in large-scale societal disruption on the island. Another study that denotes a complex dynamic between the climate and population responses is Kelly et al. ${ }^{10}$. They examine the relationship between climate and population size over the past 13,000 years at Bighorn Basic in Wyoming through the Granger causality test. Their findings suggest that, while population level is closely linked with the changes in climatic conditions, climatic changes do not immediately translate to changes in the population level. Instead, they find a persistent 100-300 year time lag between a change in the moisture level and demographic responses. These studies remind us that the relationship between climate changes and demographic responses during the Chulmun period should be conceived beyond the terms of the cause-and-effect account.

As a theoretical next-step, we suggest to emphasize the importance of the long-term view in the current discussions of Chulmun population changes. Current discussions on Chulmun population changes are often constructed in a unilinear adaptationist framework, where people are viewed as passive responders to external factors poised as the enforcing causes for cultural changes. It is undeniable that Chulmun people would have had to respond to various external impacts throughout their lives. However, such an adaptationist framework can overshadow the potential that the people's long-term history had been actively shaping their responses to the various external factors and thereby impacting their cultural trajectory in complex ways and surrounding environments - a key premise of Traditional Ecological Knowledge Theory and Niche Construction Theory ${ }^{48}$. 
This study highlights TEK as a possible factor for the resilience of the population in the coastal region as reflected in the region's relatively moderate rate of decline compared to the inland during the Late/Final phase. Our remark is based on the longue durée view of the Chulmun culture. Chulmun culture was sustained in Korea for over 6,500 years. By managing resources and knowledge that they inherited and passed down through hundreds of generations, the coastal Chulmun people may have succeeded in sustaining their traditional ways of life despite the cultural and environmental changes that they encountered during the Late and Final phases. We believe this long-term view is critical to move forward the current discourse on Chulmun population trajectories away from the simple cause-and-effect explanations.

\section{Methods}

\section{${ }^{14} \mathrm{C}$ Dates}

We collated an initial dataset comprising $894{ }^{14} \mathrm{C}$ dates from 163 archaeological sites in South Korea and analyzed a subset of 683 dates from 136 sites after removing samples with an unknown origin, ones dated on marine specimens, and those with ${ }^{14} \mathrm{C}$ age outside the bracket $6,200-2,800$. We further separated our dataset into two groups based on the sampling location of the dates: coastal $\left(\mathrm{n}_{\text {dates }}=363\right.$, $\left.\mathrm{n}_{\text {sites }}=51\right)$ when $<2 \mathrm{~km}$ from the current coast; and inland $\left(\mathrm{n}=319, \mathrm{n}_{\text {sites }}=85\right)$ when $>2 \mathrm{~km}$ from the current coast (Fig. 1). The sample also comprises 45 dates associated with broomcorn (Panicum miliaceum) or foxtail millet (Setaria italica) (Supplementary Fig. S2-S3).

\section{Age-Depth Models}

We re-fitted age-depth models (Supplementary Fig. S4-S6) on three recently published paleo-climatic proxies based on the ratio of arboreal and total pollen (AP/TP) ${ }^{29,30}$ and alkenone-derived sea surface temperature reconstruction ${ }^{31}$. For each dataset we used Bayesian nonparametric Compound PoissonGamma model ${ }^{49}$ with IntCal $20^{50}$ and Marine $20^{51}$ calibration curves, the latter with locally obtained reservoir corrections (see Supplementary materials for details). For each proxy, we visually identified (Fig. S7) the start point and the endpoint of the largest drop in the estimated sea-surface temperature ${ }^{31}\left(d_{1}\right.$ and $\left.d_{2}\right), A P / T P^{29,30}\left(f_{1}\right.$ and $f_{2} ; g_{1}$ and $g_{2}$ ) and then inferred their dates using posterior samples (Fig. S8).

\section{Summed Probability Distribution Analysis}

Probability distributions associated with calibrated ${ }^{14} \mathrm{C}$ dates were aggregated to generate summed probability distributions (SPD). In order to take into account arbitrary and idiosyncratic definitions of sites as well as to reduce the potential impact of inter-site variation in dating intensity, a spatial and temporal binning procedure was carried out before generating the curves. The former was achieved by identifying spatial clusters of archaeological sites using the DBSCAN algorithm ${ }^{52}$ with $\varepsilon=1 \mathrm{~km}$ and minPts $=1$. Temporal binning was carried out by grouping dates within the same cluster using the binPrep function of the rcarbon package ${ }^{20}$ using a temporal threshold $(h)$ set to 100 years. Dates were calibrated using the 
IntCal20 calibration curve ${ }^{50}$, and aggregated to sum to unity within each cluster-bin before summation. Three SPDs were generated, one for each region (i.e. coastal and inland sites) using the binning procedure described above and one for dates associated with millets but without spatial and temporal binning.

SPDs of coastal and inland sites were compared using a mark-permutation test ${ }^{32}$ with a time range set to 7,000-3,000 cal. BP, and with 1,000 replicates to compute the global p-value (Fig. S1). The same method and settings were used to statistically assess whether the relative proportion of $14 \mathrm{C}$ dates associated with millets $\left(\mathrm{n}_{\text {total }}=682, \mathrm{n}_{\text {millets }}=45 ; 6.59 \%\right)$ changed over time.

\section{Bayesian estimates of growth rate and change point}

We fitted a bounded double-exponential growth model ${ }^{22}$ using the nimbleCarbon package ${ }^{53}$ to estimate the growth rates and identify the start-point of the decline in the density of ${ }^{14} \mathrm{C}$ dates between 7,000 and 3,000 cal. BP. The bounded Double-Exponential growth model is defined by the following probability mass function:

$$
\begin{aligned}
& p_{t=a-i}=\frac{(1+r)^{i}}{\sum_{i=0}^{a-b}(1+r)^{i}} \\
& r= \begin{cases}r_{1}, & t>c \\
r_{2}, & t \leq c\end{cases}
\end{aligned}
$$

where $p_{t}$ is the probability of sampling a ${ }^{14} \mathrm{C}$ date from time $t$ (in cal. BP), $r$ is the growth rate (equal to $r_{1}$ ) before the change point $c$ and to $\mathrm{r} 2$ afterwards), and $a$ and $b$ are boundary parameters so that $\mathrm{p}_{\mathrm{t}<\mathrm{b}}=\mathrm{p}_{\mathrm{t}<\mathrm{a}}$ $=0$. To account for the potential impact of inter-site variation in sampling intensity we selected the ${ }^{14} \mathrm{C}$ date with the smallest error for each spatio-temporal bin, and excluded all dates with cumulative calibrated probability below 0.5 between 7,000 and $3,000 \mathrm{cal}$. BP. The resulting thinned dataset consisted of 197 dates for the coastal sites and 179 dates for the inland sites. We treated the boundary parameters as constant ( $a=7,000 \mathrm{cal}$. BP; $b=3,000 \mathrm{cal}$. BP), and used the following priors for our three parameters of interest:

$$
\begin{aligned}
& r_{1} \sim \operatorname{Normal}(\mu=0, \sigma=0.0004) \\
& r_{2} \sim \operatorname{Norma}(\mu=0, \sigma=0.0004)
\end{aligned}
$$

$c \sim$ TruncatedNormal $(a=3000, b=7000, \mu=5000, \sigma=1000)$ 
Which ensured both positive and negative growth rates within ranges typically observed in SPDs (cf Zahid et al. ${ }^{33}$ ), and a weakly informative prior for the change point with a slightly lower probability of occurrence in the boundary of the window of analysis. Prior predictive checks were carried out to examine the prior settings (Fig. S9).

Posterior samples of the parameters $r_{1}, r_{2}$, and $c$ were obtained using MCMC via the nimble package ${ }^{54,55}$, using for each data set (coastal, inland, and a combined set) three chains of 100,000 iterations, a burn-in of 10,000 steps, with the thinning parameter set to 6 . Convergence and mixing of the chains were examined using trace plots, Gelman-Rubin diagnostic ${ }^{56}$, and effective sample sizes (Fig. S11, Table S5). Posterior predictive checks were carried out by comparing the observed SPDs (using the thinned dataset) against envelopes of 500 SPDs generated from growth models based on posterior parameters (Fig. S13).

\section{Measuring temporal relation between demographic and climatic events}

The temporal order and distance between the change points in growth rates detected from the Bayesian analysis of the $14 \mathrm{C}$ dates of each data set $\left(c_{\text {inland }}, c_{\text {coastal, }}\right.$ and $\left.c_{\text {all }}\right)$ and the start point $\left(d_{1}, f_{1}\right.$, and $\left.g_{1}\right)$ and end point of the abrupt decline $\left(d_{2}, f_{2}\right.$, and $\left.g_{2}\right)$ were computed by randomly sampling 5,000 values from the posterior distribution and computing the difference in the obtained calendar years.

\section{Declarations}

\section{Data availability}

All raw data and $\mathrm{R}$ scripts can be found in a dedicated GitHub repository (https://github.com/ercrema/NeolithicKoreaDemography).

\section{Acknowledgements}

This work was funded by the Korean Studies Promotion Service/Academy of Korean Studies (Laboratory Program for Korean Studies, AKS-2015-Lab-2250001). The authors would like to thank Jungjae Park and Jinheum Park (Geography, Seoul National University) for sharing raw pollen counts on which their publications are based. E.R.C. would like to acknowledge Philip Leverhulme Prize (No. PLP-2019-304).

\section{Contributions}

E.R.C., H.K., and G.L. designed the study. H.K. and G.L. gathered and validated the data. E.R.C. performed the analysis and wrote the method section. H.K. wrote the main text except the method section. E.R.C and H.K wrote the supplementary information.

\section{Competing interests}

The authors declare no competing interests. 


\section{References}

1. Childe, V. G. The Most Ancient East (Routledge and Kegan Paul, London, 1928).

2. Braidwood, R. J. The agricultural revolution. Sci. Am, 203, 130-152 (1960).

3. Bar-Yosef, O.. The impact of the Late Pleistocene- Early Holocene climatic changes on humans in southwest Asia. in The impact of the Late Pleistocene- Early Holocene climatic changes on humans in southwest Asia (eds. Straus, L. G., Eriksen, B. V., Erlandson, J. M. \& Yesner, D. R.)61-78(New York (NY):Plenum Press, 1996).

4. Thomas, E. R. et al. The 8.2 ka event from Greenland ice cores. Q. Sci. Rev, 26, 70-81 (2007).

5. Maher, L. A., Banning, E. B. \& Chazan, M. Oasis or Mirage? Assessing the Role of Abrupt Climate Change in the Prehistory of the Southern Levant. Camb. Archaeol. J, 21, 1-29 (2011).

6. Chaput, M. \& Gajewski, K. Radiocarbon dates as estimates of ancient human population size. Anthropocene, 15, 3-12 (2016).

7. Kaufman, D. et al. A global database of Holocene paleotemperature records. Sci Data, 7, 115 (2020).

8. Rick, J. W. Dates as data: an examination of the Peruvian preceramic radiocarbon record. Am. Antiq, 52, 55-73 (1987).

9. Shennan, S. et al. Regional population collapse followed initial agriculture booms in mid-Holocene Europe. Nature Communications, 4, 2486 (2013).

10. Kelly, R. L., Surovell, T. A., Shuman, B. N. \& Smith, G. M. A continuous climatic impact on Holocene human population in the Rocky Mountains. Proceedings of the National Academy of Sciences of the United States of America, 110, 443-447 (2013).

11. Gajewski, K., Kriesche, B., Chaput, M. A., Kulik, R. \& Schmidt, V. Human-vegetation interactions during the Holocene in North America. Vegetation History and Archaeobotany, 28, 635-647 (2019).

12. Lima, M. et al. Ecology of the collapse of Rapa Nui society. Proceedings of the Royal Society B 287, 20200662(2020).

13. McLaughlin, T. R., Gómez-Puche, M., Cascalheira, J. \& Bicho, N. \& Fernández-López de Pablo, J. Late Glacial and Early Holocene human demographic responses to climatic and environmental change in Atlantic Iberia. Philosophical Transactions of the Royal Society B, 376, 20190724 (2020).

14. Fyfe, R. M. et al. Prehistoric palaeodemographics and regional land cover change in eastern lberia. The Holocene, 29, 799-815 (2019).

15. Palmisano, A., Lawrence, D., de Gruchy, M. W., Bevan, A. \& Shennan, S. Holocene regional population dynamics and climatic trends in the Near East: A first comparison using archaeo-demographic proxies. Q. Sci. Rev, 252, 106739 (2021).

16. Stewart, M., Carleton, W. C. \& Groucutt, H. S. Climate change, not human population growth, correlates with Late Quaternary megafauna declines in North America. Nature Communications, 12, 965 (2021). 
17. Williams, A. N. The use of summed radiocarbon probability distributions in archaeology: a review of methods. Journal of Archaeological Science, 39, 578-589 (2012).

18. Surovell, T. A. \& Brantingham, P. J. A note on the use of temporal frequency distributions in studies of prehistoric demography. Journal of Archaeological Science, 34, 1868-1877 (2007).

19. Carleton, W. C. \& Groucutt, H. S. Sum things are not what they seem: Problems with point-wise interpretations and quantitative analyses of proxies based on aggregated radiocarbon dates. The Holocene, 31, 630-643 (2021).

20. Crema, E. R., Bevan, A. \& INFERENCE FROM LARGE SETS OF RADIOCARBON DATES: SOFTWARE AND METHODS., 63, 23-39 (2021).

21. Timpson, A., Barberena, R., Thomas, M. G., Méndez, C. \& Manning, K. Directly modelling population dynamics in the South American Arid Diagonal using $14 \mathrm{C}$ dates. Philosophical Transactions of the Royal Society B: Biological Sciences, 376, 20190723 (2021).

22. Crema, E. R. \& Shoda, S. A Bayesian approach for fitting and comparing demographic growth models of radiocarbon dates: A case study on the Jomon-Yayoi transition in Kyushu (Japan). PLOS ONE, 16, e0251695 (2021).

23. Bae, K., Bae, C. J. \& Kim, J. C. Reconstructing Human Subsistence Strategies During the Korean Neolithic: Contributions from Zooarchaeology, Geosciences, and Radiocarbon Dating., 55, 13501357 (2013).

24. Ahn, S. M., Kim, J. S. \& Hwang, J. H. Sedentism, Settlements, and Radiocarbon Dates of Neolithic Korea. Asian Perspect, 54, 113-143 (2015).

25. Oh, Y. J. et al. USING RADIOCARBON DATES AS A PROXY FOR POPULATION CHANGE., 59, 17611770 (2017).

26. Ahn, S. M. \& Hwang, J. H. Temporal fluctuation of human occupation during the 7th-3rd millennium cal BP in the central-western Korean Peninsula. Quatern. Int, 384, 28-36 (2015).

27. Binford, L. R. Willow Smoke and Dogs' Tails: Hunter-Gatherer Settlement Systems and Archaeological Site Formation. Am. Antiq, 45, 4-20 (1980).

28. Shin, S. C., Rhee, S. N. \& Aikens, C. M. Chulmun Neolithic Intensification, Complexity, and Emerging Agriculture in Korea. Asian Perspect, 51, 68-109 (2012).

29. Park, J. J. et al. Abrupt Holocene climate shifts in coastal East Asia, including the $8.2 \mathrm{ka}, 4.2 \mathrm{ka}$, and 2.8 ka BP events, and societal responses on the Korean peninsula. Sci. Rep, 9, 10806 (2019).

30. Constantine, M., Kim, M. K. \& Park, J. J. Mid- to late Holocene cooling events in the Korean Peninsula and their possible impact on ancient societies. Quatern. Res, 92, 98-108 (2019).

31. Kim, J. H. et al. North Pacific and North Atlantic sea-surface temperature variability during the Holocene. Q. Sci. Rev, 23, 2141-2154 (2004).

32. Crema, E. R., Habu, J., Kobayashi, K. \& Madella, M. Summed Probability Distribution of 14C Dates Suggests Regional Divergences in the Population Dynamics of the Jomon Period in Eastern Japan. PLOS ONE, 11, e0154809 (2016). 
33. Zahid, H. J., Robinson, E. \& Kelly, R. L. Agriculture, population growth, and statistical analysis of the radiocarbon record. Proceedings of the National Academy of Sciences of the United States of America, 113, 931-935 (2016).

34. Crema, E. R. \& Kobayashi, K. A multi-proxy inference of Jōmon population dynamics using bayesian phase models, residential data, and summed probability distribution of $14 \mathrm{C}$ dates. Journal of Archaeological Science, 117, 105136 (2020).

35. Jo, J. Y. Dryfield Farming (Hyangmunsa, Seoul, 1995).

36. Kaume, R. N. \& Panicum miliaceum, L.. Record from Protabase. in PROTA (Plant Resources of Tropical Africa / Ressources végétales de l'Afrique tropicale) (eds. Brink, M. \& Belay, G.) (Wageningen, Netherlands, 2006).

37. Brink, M. Setaria italica (L.) P. Beauv. Record from Protabase. in PROTA (Plant Resources of Tropical Africa / Ressources végétales de l'Afrique tropicale) (eds. Brink, M. \& Belay, G.) (Wageningen, Netherlands, 2006).

38. Lee, G. A.. The spread of domesticated plant resources in prehistoric northeast Asia. in Routledge Handbook of Archaeology and Globalization (ed. Hodos, T.)394-412(Taylor \& Francis/Routledge Press, 2016).

39. Zhao, Z. New Archaeobotanic Data for the Study of the Origins of Agriculture in China. Curr. Anthropol, 52, S295-S306 (2011).

40. Park, G. T. A Study on the Neolithic Culture of Jeju Island (Dept. of Archaeology, Pusan National University, 2021).

41. Lee, G. A. The Chulmun Period of Korea: Current Findings and Discourse on Korean Neolithic Culture. in Handbook of East and Southeast Asian Archaeology (eds. Habu, J., Lape, P. V. \& Olsen, J. W.) 451481(Springer New York, 2017). doi:10.1007/978-1-4939-6521-2_28.

42. Lee, G. A., Cho, M. S. \& Obata, H. Coastal farmers during the Early and Middle Holocene: Archaeobotanical and grain impression studies on the east coast Korea. Quatern. Int, 529, 57-74 (2019).

43. Kwak, S. K., Obata, H. \& Lee, G. A. Broad-spectrum foodways in southern coastal Korea in the Holocene: Isotopic and archaeobotanical signatures in Neolithic shell middens. The Journal of Island and Coastal Archaeology, 1-29, https://doi.org/10.1080/15564894.2020.1776427 (2020).

44. National Museum of Korea. Amsadong II (National Museum of Korea, 1999).

45. Kim, J. S. Land-Use Conflict and the Rate of the Transition to Agricultural Economy: A Comparative Study of Southern Scandinavia and Central-Western Korea. Journal of Archaeological Method and Theory, 10, 277-321 (2003).

46. Berkes, F. Sacred ecology (Routledge, New York, 2008).

47. DiNapoli, R. J., Crema, E. R., Lipo, C. P., Rieth, T. M. \& Hunt, T. L. Approximate Bayesian Computation of radiocarbon and paleoenvironmental record shows population resilience on Rapa Nui (Easter Island). Nature Communications, 12, 3939 (2021). 
48. Laland, K., Matthews, B. \& Feldman, M. W. An introduction to niche construction theory. Evol. Ecol, 30, 191-202 (2016).

49. Haslett, J. \& Parnell, A. A simple monotone process with application to radiocarbon-dated depth chronologies. Journal of the Royal Statistical Society: Series C (Applied Statistics), 57, 399-418 (2008).

50. Reimer, P. J. et al. The IntCal20 Northern Hemisphere Radiocarbon Age Calibration Curve (0-55 cal kBP)., 62, 725-757 (2020).

51. Heaton, T. J. et al. Marine20-The Marine Radiocarbon Age Calibration Curve (0-55,000 cal BP)., 62, 779-820 (2020).

52. Ester, M., Kriegel, H. P., Sander, J., Xu, X. A. \& Density-Based Algorithm for Discovering Clusters in Large Spatial Databases with Noise. in Proceedings of 2nd International Conference on Knowledge Discovery and Data Mining (KDD-96) (eds. Simoudis, E., Han, J. \& Fayyad, U.) 226-231 (Portland: AAAI Press 1996).

53. Crema, E. R. \& nimbleCarbon Models and Utility Functions for Bayesian Analysis of Radiocarbon Dates with NIMBLE (v.0.1.2)(2021).

54. de Valpine, P. et al. Programming With Models: Writing Statistical Algorithms for General Model Structures With NIMBLE. Journal of Computational and Graphical Statistics, 26, 403-413 (2017).

55. de Valpine, P. et al. NIMBLE: MCMC, Particle Filtering, and Programmable Hierarchical Modeling. 2021. Available: https://doi.org/10.5281/zenodo.1211190

56. Gelman, A. \& Rubin, D. B. Inference from Iterative Simulation Using Multiple Sequences. Statistical Science, 7, 457-472 (1992).

\section{Figures}


a

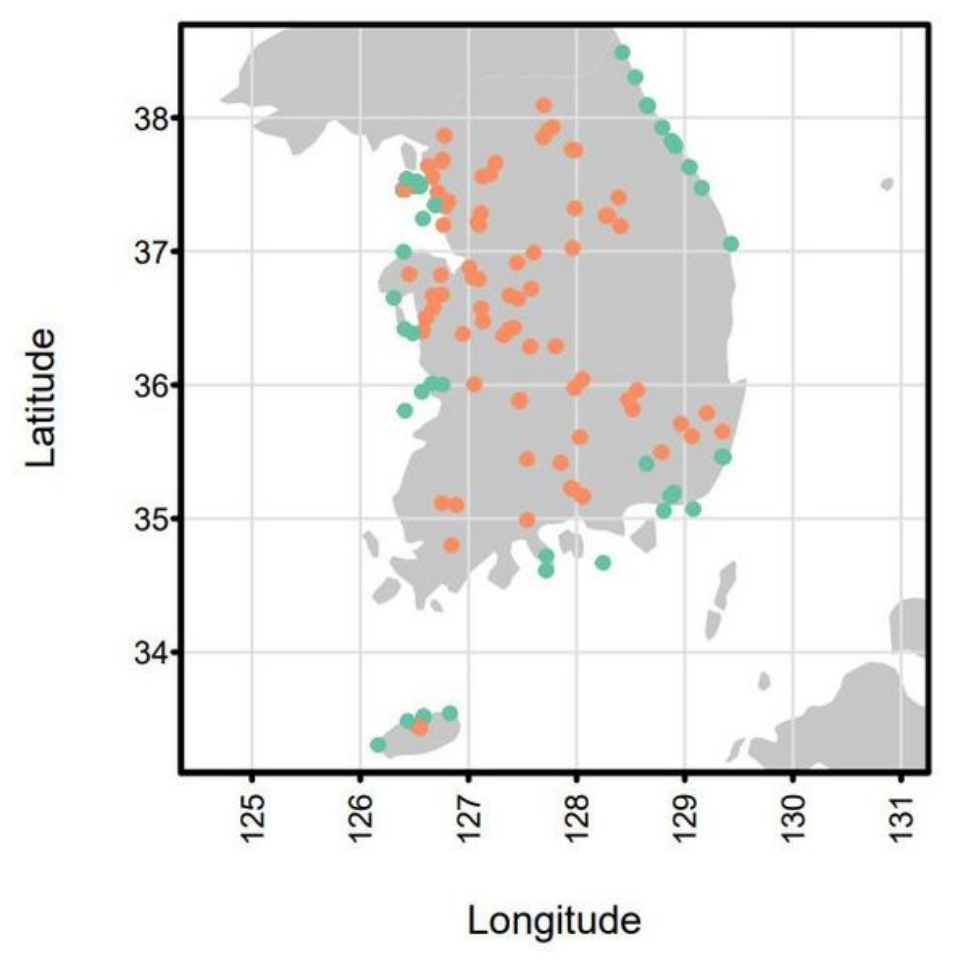

b

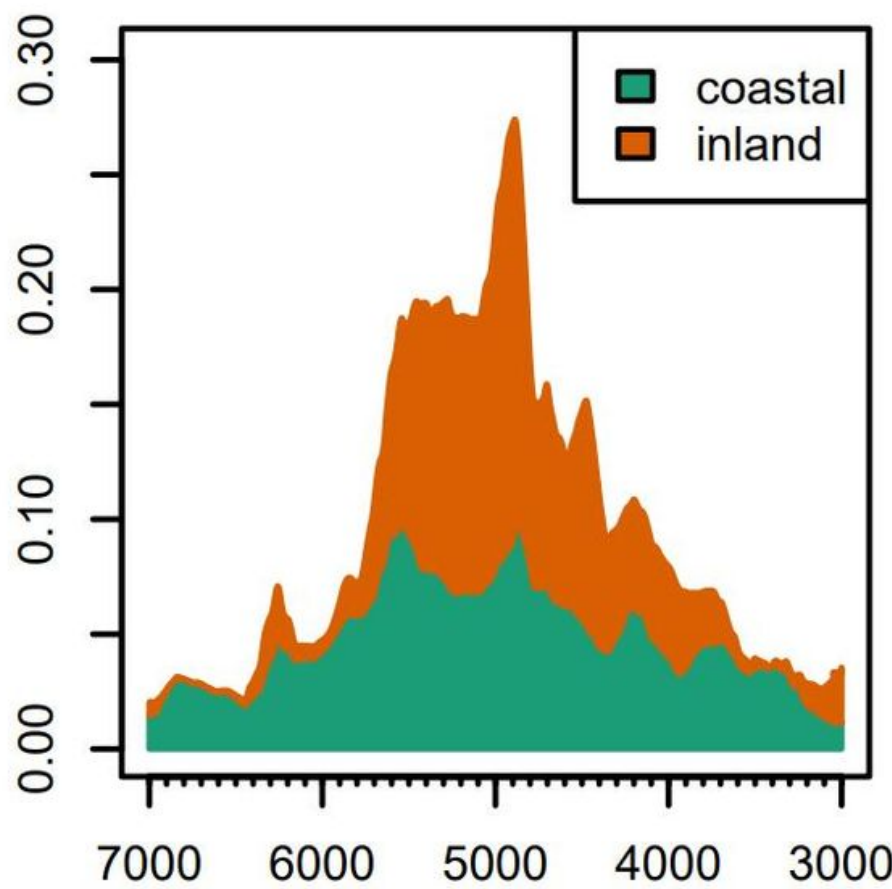

Years cal BP

Figure 1

Distribution of sampling location of $14 \mathrm{C}$ dates (a) and summed probability distribution of the coastal and inland regions (b).
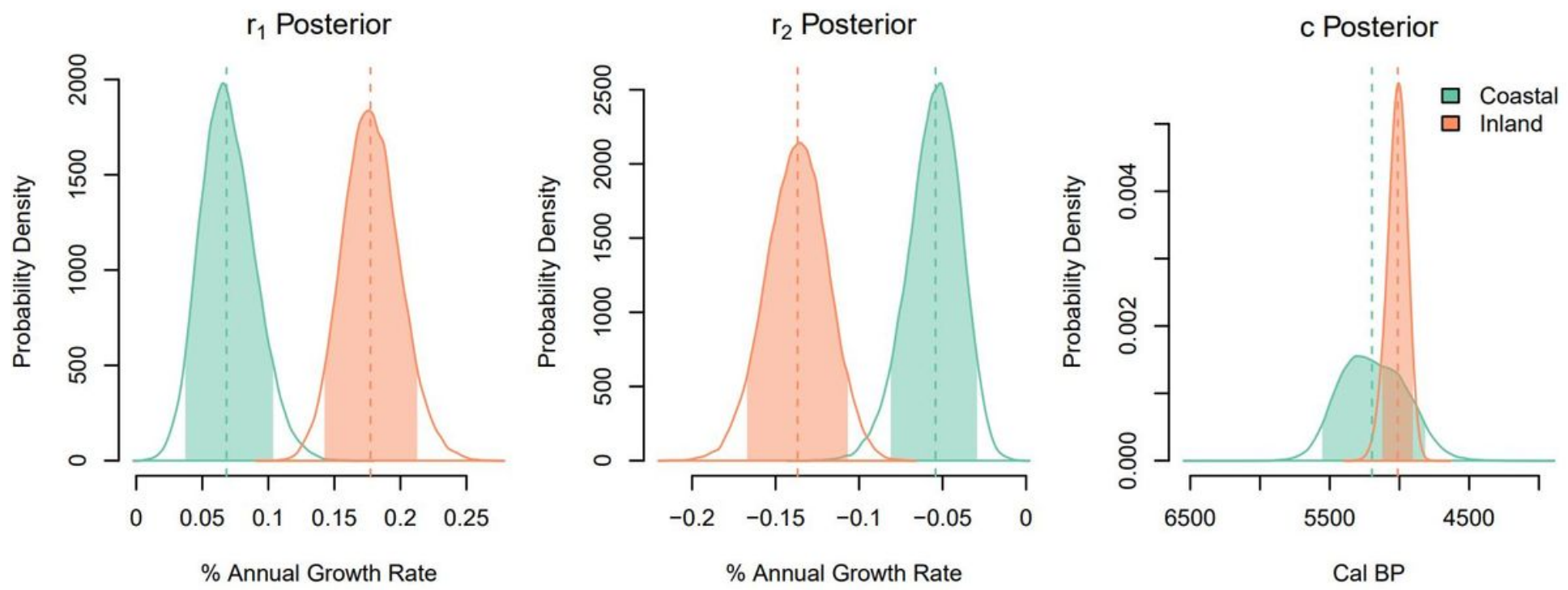

Figure 2

Marginal posterior distribution of growth rates $\mathrm{r} 1$ and $\mathrm{r} 2$ and change-point $\mathrm{c}$ for the inland and coastal radiocarbon dates. Highlighted regions depict the $90 \%$ higher posterior density interval. 

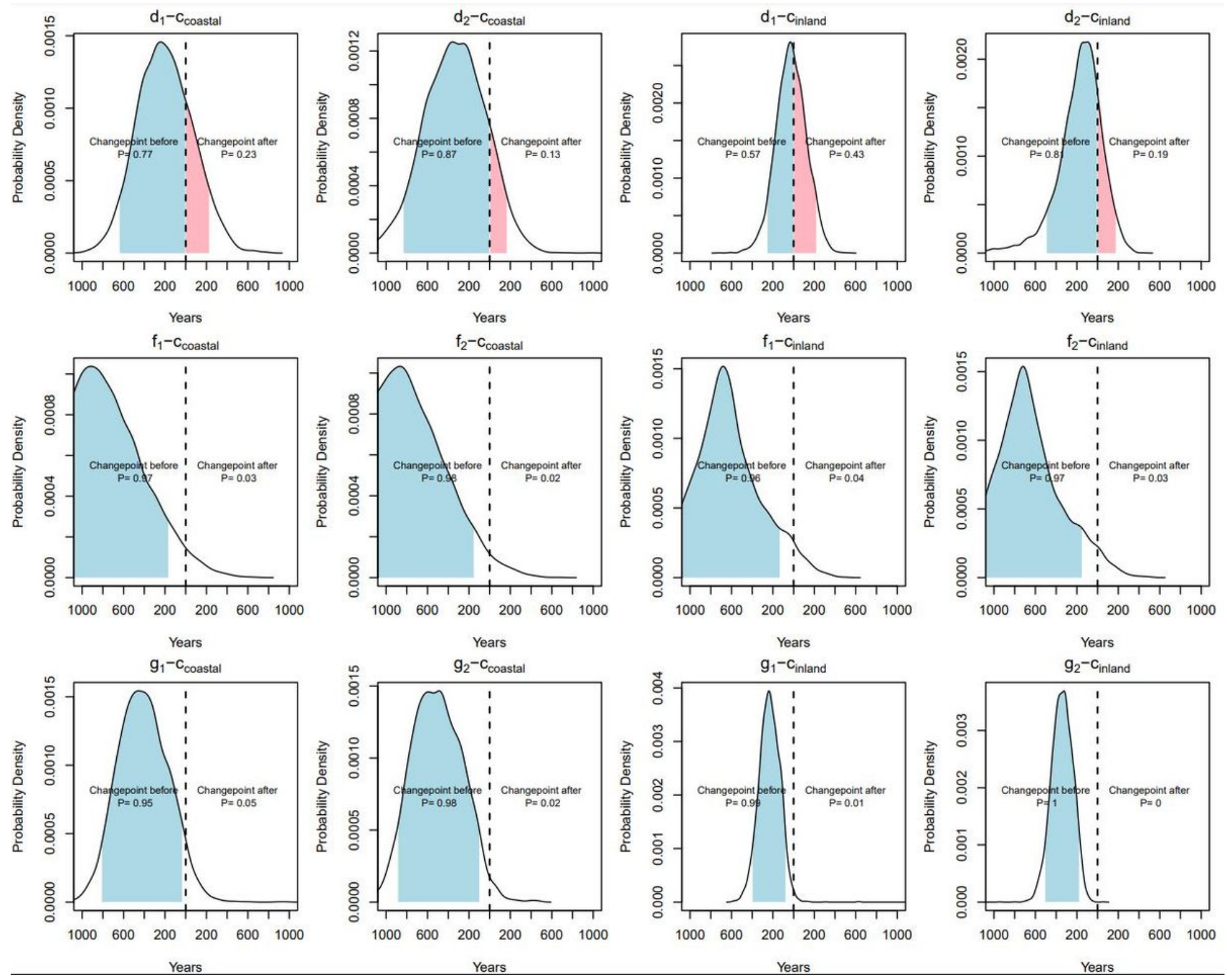

\section{Figure 3}

Temporal relationships between $14 \mathrm{C}$ density change points in in coastal (columns 1,2) and inland regions (columns 3,4 ) and climatic events based on drops in alkenone-derived sea-surface temperature from Kim et al.31 (d1, d2); and drops in arboreal to total pollen (AP/TP) ratios from Constantine et al.30 $(f 1, f 2)$ and Park et al.29 (g1, g2). Subscript numbers represent the start (1) and endpoint (2) of the decline. See ESM for details. 


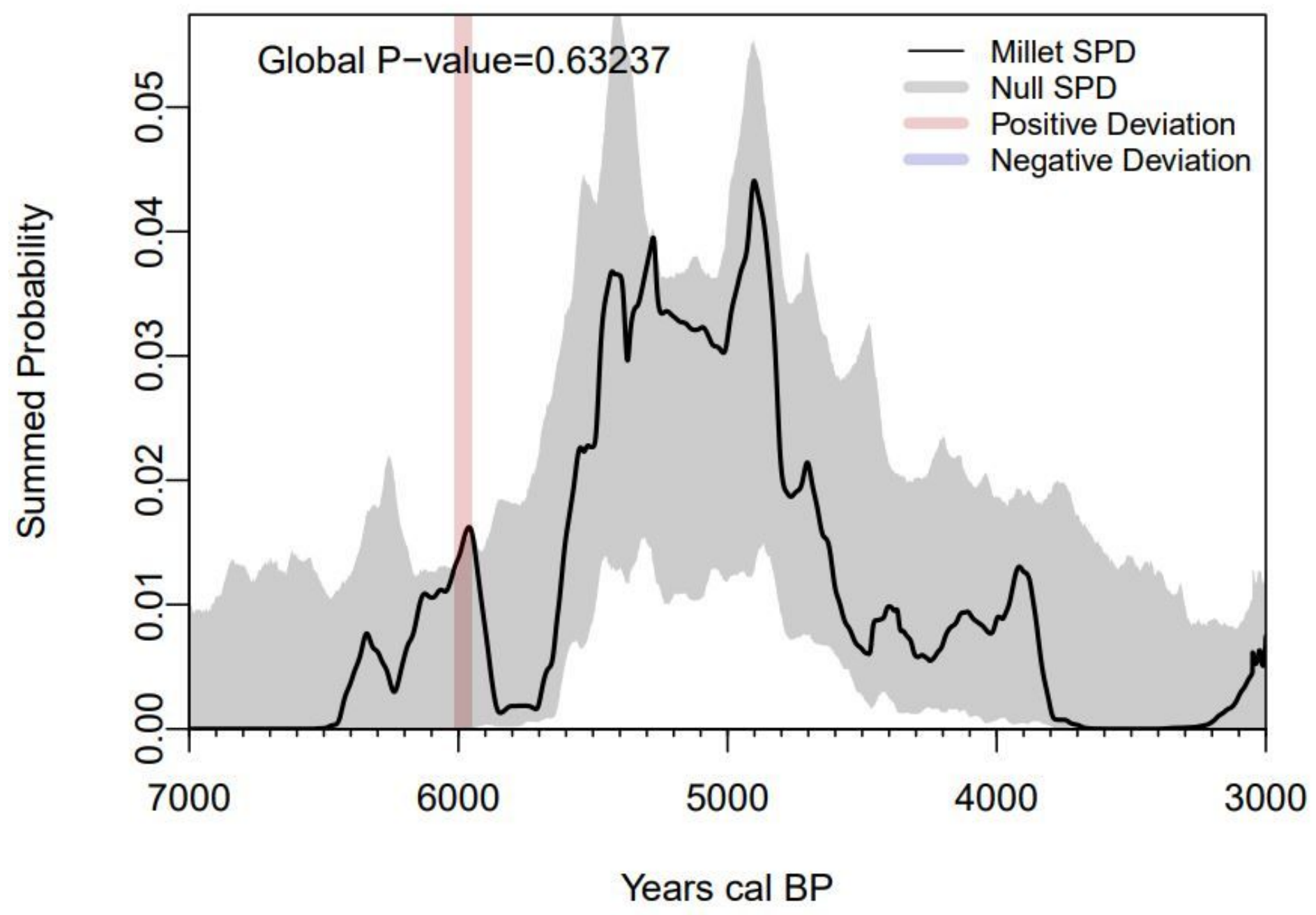

Figure 4

Mark permutation test of millet vs combined 14C dates from both regions

\section{Supplementary Files}

This is a list of supplementary files associated with this preprint. Click to download.

- SIKimetal.pdf 